INPLASY

PROTOCOL

To cite: Wang et al. The Natural Orifice Specimen Extraction Surgery Compared With Conventional Laparoscopy for Colorectal Cancer: A Meta-analysis of Efficacy and Long-term oncological Outcomes. Inplasy protocol 202170055. doi: 10.37766/inplasy2021.7.0055

Received: 17 July 2021

Published: 17 July 2021

Corresponding author: Zhen Li

doctorlz79@163.com

Author Affiliation:

The First Affiliated Hospital of Zhengzhou University.

Support: No funding.

Review Stage at time of this submission: Preliminary searches.

Conflicts of interest: None declared.

\section{The Natural Orifice Specimen Extraction Surgery Compared With Conventional Laparoscopy for Colorectal Cancer: A Meta-analysis of Efficacy and Long-term oncological Outcomes}

Wang, S1; Tang, J2; Sun, W3; Yao, H4; Li, Z5.

Review question / Objective: (1) Participants: colorectal cancer patients undergoing surgery. (2) Intervention: laparoscopic radical colorectal resection and specimen extraction via the anus or vagina. (3) Comparison: Comparing therapeutic effects of NOSES and conventional laparoscopic surgery (specimen extraction through the abdominal wall) in patients. (4) Outcome: postoperative complications, the time of first exhaust, length of hospital stay, pelvic floor function, cosmetic result, operative time, intraoperative blood loss, lymph nodes harvested, visual analogue scale score(VAS) in postoperative day 1 (POD 1), additional analgesics usage, 3years disease-free survival, and 3-years overall survival. (5) Study design: published randomized controlled trials, nonrandomized controlled trials, and retrospective studies.

INPLASY registration number: This protocol was registered with the International Platform of Registered Systematic Review and Meta-Analysis Protocols (INPLASY) on 17 July 2021 and was last updated on 17 July 2021 (registration number INPLASY202170055).

\section{INTRODUCTION}

Review question / Objective: (1) Participants: colorectal cancer patients undergoing surgery. (2) Intervention: laparoscopic radical colorectal resection and specimen extraction via the anus or vagina. (3) Comparison: Comparing therapeutic effects of NOSES and conventional laparoscopic surgery (specimen extraction through the abdominal wall) in patients. (4) Outcome: postoperative complications, the time of first exhaust, length of hospital stay, pelvic floor function, cosmetic result, operative time, intraoperative blood loss, lymph 
nodes harvested, visual analogue scale score(VAS) in postoperative day 1 (POD 1), additional analgesics usage, 3-years disease-free survival, and 3-years overall survival. (5) Study design: published randomized controlled trials, nonrandomized controlled trials, and retrospective studies.

Condition being studied: Colorectal cancer (CRC) is the third most commonly diagnosed malignancy and the second leading cause of cancer death worldwide, which imposes a tremendous medical and economic burden. Currently, surgical resection is the most effective treatment for CRC. Since Jacobs reported laparoscopic colorectal cancer resection firstly, the operative approach to treating colorectal neoplasms has shifted to minimally invasive surgery. Most studies have confirmed that laparoscopic colorectal cancer has similar results of radical resection and long-term outcome compared with open surgery, which had significant advantages in reducing the incidence of postoperative complications, relieving pain, and accelerating recovery. However, conventional laparoscopic surgery requires an abdominal incision (approximately 4-8cm) to extract specimen, dramatically increasing the incidence of incision-related complications such as abdominal incision infection, wound fat liquefaction, incisional hernia. Franklin reported laparoscopic sigmoid resection with transrectal specimen extraction. This technique had attracted much attention. Natural Orifice Specimen Extraction Surgery (NOSES) has been picked up nowadays, a technique for extracting specimens from natural orifices(such as rectum, anus, or vagina). This technique does not require an additional abdominal incision to complete intraperitoneal anastomosis as compared to conventional laparoscopy. Nevertheless, some scholars believed that NOSES increased the risk of intra-abdominal infection and tumor cell implantation, challenging this technique's safety. It is unclear that current conclusion on the effect of NOSES in reducing postoperative complications and oncological safety. we performed a meta- analysis to provide support from high-level evidence-based medicine for NOSES in the treatment of colorectal cancer.

\section{METHODS}

Search strategy: The "Web of Science", "Embase", "PubMed", "Cochrane Library", and "EBSCO" databases were systematically searched to retrieve relevant articles published up to May 2021. The search included the following keywords: "Colorectal Neoplasms", "Colorectal Tumors", " Colorectal Cancers" "Colorectal Carcinomas", "natural orifice specimen extraction surgery", "transvaginal specimen extraction", "transanal specimen extraction" and "Laparoscopy".

Participant or population: Colorectal cancer patients undergoing surgery.

Intervention: Laparoscopic radical colorectal resection and specimen extraction via the anus or vagina.

Comparator: Comparing therapeutic effects of NOSES and conventional laparoscopic surgery (specimen extraction through the abdominal wall) in patients.

Study designs to be included: Randomized controlled trials, non-randomized controlled trials, and retrospective studies.

Eligibility criteria: (1) Participants: colorectal cancer patients undergoing surgery. (2) Intervention: laparoscopic radical colorectal resection and specimen extraction via the anus or vagina. (3) Comparison: Comparing therapeutic effects of NOSES and conventional laparoscopic surgery (specimen extraction through the abdominal wall) in patients. (4) Outcome: postoperative complications, the time of first exhaust, length of hospital stay, pelvic floor function, cosmetic result, operative time, intraoperative blood loss, lymph nodes harvested, visual analogue scale score(VAS) in postoperative day 1 (POD 1), additional analgesics usage, 3years disease-free survival, and 3-years overall survival. (5) Study design: published randomized controlled trials, non- 
randomized controlled trials, and retrospective studies, the language was limited to English.

Information sources: The "Web of Science", "Embase", "PubMed", "Cochrane Library", and "EBSCO" databases.

Main outcome(s): Postoperative complications, 3-years disease-free survival, and 3-years overall survival.

Additional outcome(s): The time of first exhaust, length of hospital stay, pelvic floor function, cosmetic result, operative time, intraoperative blood loss, lymph nodes harvested, visual analogue scale score(VAS) in postoperative day 1 (POD 1), additional analgesics usage.

Data management: Extracted data are exported to STATA 14 statistical software for analysis.Data extraction and literature evaluation are independently completed by two researchers. Any inconsistencies between the two researchers are resolved through consultation of the full text.

Quality assessment / Risk of bias analysis: The Cochrane Collaborations tool for evaluating the risk of bias in randomized controlled trials(RCTs). Moreover, the Newcastle Ottawa scale (NOS) for assessing the quality of non-randomized controlled studies and retrospective studies.

Strategy of data synthesis: Weighted/ Standardized MD (WMD/SMD) and Odds Ratio (OR) are used to analyze the continuous variables and dichotomous variables, respectively. If the mean and variance cannot be available from studies, they are derived from the median values and ranges using the method described by Hozo et al. If a study do not provide the hazard ratio (HR) and $95 \%$ Cls of 3 -year disease-free survival and 3-year overall survival, we will use the method provided by Tierney et al to calculate the HR and 95\% Cls from the survival curve.
Subgroup analysis: Subgroup analysis is performed according to different specimen extraction methods.

Sensitivity analysis: When the heterogeneity is very large $(12>75 \%)$, sensitivity analysis is performed to assess the stability of the results.

Language: The language is limited to English.

Country(ies) involved: China.

Keywords: Natural Orifice Specimen Extraction Surgery; minimally invasive surgery; Laparoscopic surgery; Colorectal cancer; Meta-analysis.

Contributions of each author:

Author 1 - Shihao Wang - The author was responsible for the research design, drafted the manuscript.

Author 2 - Jiyan Tang - The author contributed to data verification, approach evaluation, and assisted with writing the manuscript.

Author 3 - Weipeng Sun - The author participated in planning and writing the paper.

Author 4 - Hongyu Yao - The author read, provided feedback and approved the final manuscript participated in planning and writing the paper.

Author 5 - Zhen Li - The author is the corresponding author, he contributed to revision, final read and approved the manuscript. 